\title{
Advances in small bowel transplantation
}

\author{
Alp Gürkan ${ }^{1,2}$
}

Cite this paper as:

Gürkan A. Advances in small

bowel transplantation. Turk J Surg 2017; 33: 135-141.

'Department of General Surgery, Camlica Medicana Hospital,

İstanbul, Turkey

${ }^{2}$ Department of General Surgery, İstanbul Aydın University School of Medicine, İstanbul, Turkey

\footnotetext{
Address for Correspondence Alp Gürkan e-mail: alpgurkan1@gmail.com

Received: 07.03.2016 Accepted: 17.10 .2016

CCopyright 2017 by Turkish Surgical Association

Available online at www.turkjsurg.com
}

Small bowel transplantation is a life-saving surgery for patients with intestinal failure. The biggest problem in intestinal transplantation is graft rejection. Graft rejection is the main reason for morbidity and mortality. Rejection has a negative effect on the survival of the graft. While $50 \%-75 \%$ of small bowel transplantation patients experience acute rejection, chronic rejection occurs in approximately $15 \%$ of patients. Immune monitoring is crucial after small bowel transplantation. Unlike other types of transplantation, there are no non-invasive or reliable markers to predict rejection in small bowel transplantation. The diagnosis of AR is confirmed by clinical symptoms, endoscopic appearance, and pathological specimens taken by endoscopy. Thus, histopathological examinations obtained by protocol biopsies remain as the gold standard for intestinal graft monitoring; however, biopsies have some complications, especially in small grafts. In addition to the high complication rate, biopsies are non-diagnostic; thus, multiple biopsies should be performed to exclude rejection. Therefore, auxiliary assays, such as measurements of citrulline and calprotectin in the blood, cytofluorographic examination of peripheral blood immune cells, cytokine profiling, and distinct gene-set-change measurements, are increasingly being used in small bowel transplantation. Developments in the understanding of genes seem to be promising that limited gene sets, taken from blood or from intestinal biopsies, will enhance pathological diagnosis. Bone marrow mesenchymal stem cell transplantation with SBT and tissue engineering are also promising procedures.

Keywords: Small bowel transplantation, intestinal transplantation, stem cell transplantation, small bowel insufiency, short bowel syndrome

\section{INTRODUCTION}

Small bowel transplantation (SBT) has developed slower and has been performed less than other solid organ transplantations. However, it is the most effective and life-saving procedure for patients with intestinal failure who develop complications related to parenteral nutrition. The annual number of SBTs is less than that of all other types of solid organ transplantations. Although the number of SBTs has been decreasing in the United States since 2007, because of bowel rehabilitation programs and recent developments in surgical techniques such as tapering enteroplasties, the number of SBTs has substantially increased in the last 5 years in Europe, China, and Japan (1). It is estimated that 2 or 3 persons per million per year experience intestinal failure, and $15 \%$ of them become candidates for SBT (2). The mortality rate is around $40 \%$ in 5 years in patients having less than $50 \mathrm{~cm}$ of healthy small bowel remaining. Infections and/or thrombosis of vessels and liver disease developing within 2 years after SBT are the most common reasons of mortality.

Small bowel transplantation is a sophisticated procedure for patients with desperate clinical conditions. It covers a number of surgical procedures depending on organs to be transplanted, but the main procedure is the transplantation of the small bowel. Although there are different classifications for the procedure, the most common one classifies it into four groups, according to the inclusion of the liver and/or the stomach in the graft: isolated, liver-intestinal, multivisceral, and modified multivisceral transplantation (3). Although combined liver and SBT used to be the most common procedure in the past, the rate this type of SBT has decreased from $68 \%$ to $39 \%$ from 2007 to 2011 . The number of isolated small bowel transplantations has been increasing due to early referral to the transplantation units (4). SBT patients need more intense immune suppression protocols than other solid organ transplantation patients due to large size of the graft and the strong immune response usually evoked. Thus, opportunistic infections and neoplastic diseases are seen more commonly in SBT than in other solid organ transplantations. Besides, because of the large size of tissue transplanted, graft versus host disease (GVHD) is also more common in SBT than in other solid organ transplantations.

Currently, patients who experience complications in parenteral nutritional therapy are candidates for SBT. Nowadays, SBT is not indicated for patients who are dependent on parenteral nutrition, having 
no complications. Indications for STB are thrombosis of two of the six major venous accesses, episodes of catheter-related infections (two or more per year, fungemia, shock, or respiratory failure), liver disease, alterations of growth and development in children, and refractory electrolyte changes.

\section{CLINICAL AND RESEARCH CONSEQUENCES}

\section{Surgical Techniques}

Some other abdominal organs can be transplanted with a small intestinal graft, depending on the pathology. Patients with mild liver disease, revealing no signs of portal hypertension and mild hepatic fibrosis on liver biopsy, may receive an isolated intestinal graft and do not need a liver graft transplantation. On occurrence of dysmotility of the foregut with apparent problems, the stomach can be included in the graft. The preferred technique is harvesting and implanting the liver, duodenum, head of pancreas, and small bowel with bile ducts en bloc as a composite graft without damaging the vascular or other structural connections of the organs. The organs can also be harvested from the donor separately and transplanted individually, which is known as non-composite combined liver and SBT. The organs to be transplanted are decided according the underlying disease, presence and severity of liver disease, condition of other organs, and the number of previous abdominal surgeries. An isolated small bowel graft (Figure 1) is indicated in the patients with intestinal failure without a severe hepatic dysfunction. The severity and reversibility of the liver disease is diagnosed by liver biopsy. A recent study claimed that bilirubin levels, platelet count, and albumin levels in children receiving parenteral nutrition show a good correlation with the severity of the hepatic disease (5). Occurrence of bridging fibrosis or cirrhosis indicates that the liver should be added to the intestinal graft. In order to harvest the liver and intestine en bloc, the pancreaticoduodenal arc should be included in the graft. This avoids the dissection of hilar structures, which can be difficult in a donor of pediatric age. Alternatively, liver and intestine can be implanted separately. Thus, in case of severe rejection, the intestinal graft can be explanted easily without removing the hepatic graft. However, separate grafts require multiple vascular anastomosis and biliary reconstruction which cause higher risks of complications.

The superior mesenteric artery is anastomosed to the aorta. Venous anastomosis is performed between the superior mesenteric vein and the inferior vena cava (Figure 2) or the mesenteric portal system. Anastomosis to the portal system is more physiologic and has some possible immunologic advantages, but accessing the recipient's mesenteric portal system is technically more challenging than systemic drainage. In patients with mild portal hypertension with low platelet counts, absence of gastroesophageal varices, and intrahepatic cholestasis with moderate splenic enlargement, venous drainage of the isolated small bowel can be performed into the vena cava. Although the cumulative episodes of infection were higher in patients with drainage into vena cava where the protective effect of the liver is by-passed, a survival difference could not be achieved (6). In practice, anastomosis to the mesenteric superior vein is seldom associated with major problems in terms of outcome; therefore, it is performed more often because of technical simplicity. To observe the bowel endoscopically and obtain biopsies facilitating the diagnosis of rejection and per-

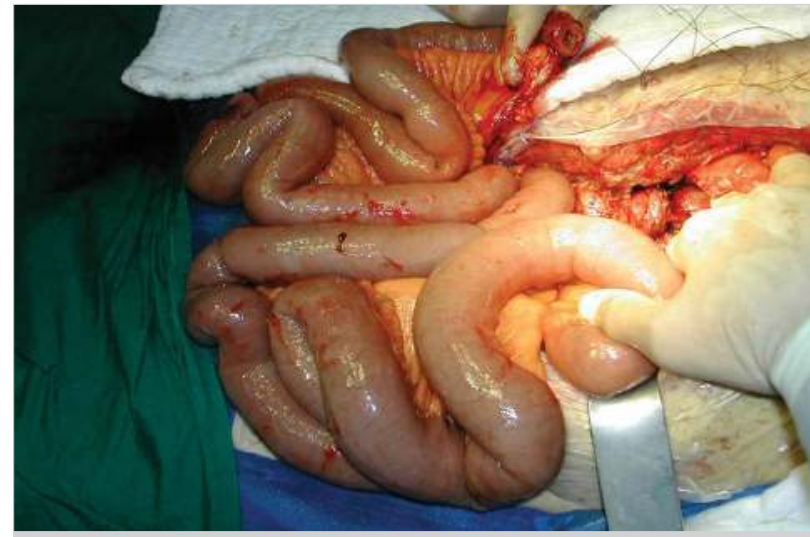

Figure 1. Isolated intestinal graft

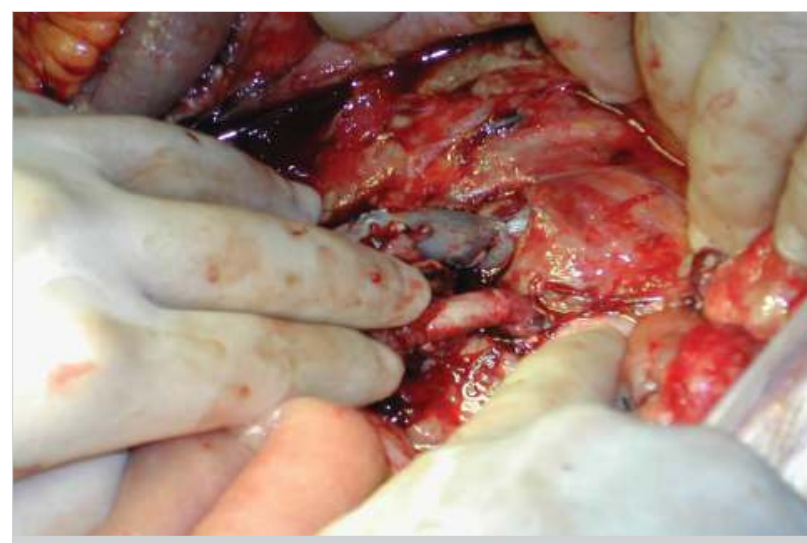

Figure 2. Mesenteric venous and arterial anastomosis of the small intestinal graft

Pediatric patients more commonly require combined liver and SBT compared with adults, due to irreversible liver damage, which is more often seen in the pediatric population under parenteral nutrition. However, both grafts are scarcely available for pediatric patients due to size problems. According to the US data, $74 \%$ of patients for intestinal transplantation need the combined liver and intestinal graft transplantation (7). Progression of the allocation system and early referral to SBT can help solve this problem.

Inclusion of the colon and spleen to intestinal grafts is controversial. Patients who receive an intestinal graft without the ileocecal valve usually do not have well-formed stools and are more likely to become dehydrated. It was thought that inclusion of the colon in small intestine grafts increases death rates and the risk of graft failure; therefore, this was previously avoided. However, recent studies have shown that inclusion of the colon does not increase morbidity, mortality, and bloodstream infections, and has benefits especially in pediatric patients (8).

Patient's liver, spleen, and pancreaticoduodenal complex should be preserved whenever possible. Different modifications can be applied for patients who need multivisceral transplantations with intact hepatic functions, especially those with Gardner and pseudo-obstruction syndromes. Sparing the native spleen also has potential advantage of reduced risk of post-transplant lymphoproliferative disorders. After showing the beneficial effect of spleen transplantation in promoting 
tolerance in animal experiments, a recent research demonstrated that adding the spleen to the multivisceral transplantation graft yielded better outcomes in terms of low acute rejection (AR), without altering the incidence of $\operatorname{GVHD}(9,10)$. Implantation of the donor spleen with liver-intestine graft is advantageous with reduced infection rate and enhanced mixed chimerism. Harvesting the distal esophagus within the multivisceral graft simplifies the transplantation procedure of foregut organs.

Although microendoscopy is not widely used, it helps visualize the transplant serosal surface and monitor mucosal blood flow of the graft during the surgery. This method is also found to be very sensitive during the postoperative and intraoperative period (11). Thus, this method helps assess the viability of the graft. However, the procedure is technically demanding and not easy to perform in a duration of 1 or 2 hours.

Loss of abdominal flexibility in patients with SBT due to massive adhesions related to multiple prior abdominal surgeries, shortage of appropriate recipient size-matched donors especially in pediatric age, scars on the abdominal wall due to fistulas, ostomies, and edema after reperfusion of the graft make the primary abdominal wall closure straitening. A primary tension-free closure of the abdominal wall is achievable in $50 \%-65 \%$ of patients (12). Although sometimes reduced-size grafts can be used to facilitate primary closure, various strategies have been introduced to reconstruct and enhance the abdominal domain. Some strategies that have been employed are usage of tissue expanders, staged abdominal closure with mesh, bioengineered skin equivalents, acellular dermal matrix, vascularized or nonvascularized rectus muscle fascia grafts, skin grafts, and vascularized abdominal wall transplantation from the same donor $(12,13)$. Abdominal wall transplantation permits primary skin and abdominal wall closure without causing abdominal compartment syndrome. However, it has some disadvantages such as necessity of a complicated vascular anastomosis, longer surgery time, and higher morbidity rate. The use of an avascular rectus allofascia is also reported with good results (14).

\section{Donor Preparation}

Donors ideally should be younger than 55 years and under no or low dose of vasoactive drugs (below $5 \mu \mathrm{gr} / \mathrm{kg} / \mathrm{min}$ of dopamine) abdominal domain of the patients with intestinal failure is usually retracted; therefore, they need smaller donors $(30 \%$ to $40 \%$ ). Owing to the development of potent drugs for the prophylaxis and treatment of cytomegalovirus, cytomegalovirus-seropositive donors are accepted, excluding receivers with negative serology. Gastrointestinal tract decontamination and utilization of antibodies for donor lymphocytes have no beneficial effect on prevention of infection, rejection episodes, or incidences of GVHD. These donors are also suitable for harvesting liver and pancreatic grafts. As these grafts share the same bloodstream, it is challenging to simultaneously harvest the grafts, but it is possible to perform the surgery without injuring the grafts.

The mucosa of the small bowel is very sensitive to ischemic injury. Infection-related mortality rate is higher and the absorptive function is lower if the intestinal graft is taken from nonheart beating donors (NHBDs). The viability of the cells may be determined by histological examinations, and thus, grafts taken from NHBDs may be used if the cells are determined to be viable. An experimental study showed that NHBDs are suitable for SBT (15).

Living donor SBT is a relatively recent type of transplantation, which is suitable especially for children with small bowel failure in whom acute decompensated hepatic failure occurs. To decrease the morbidity and mortality while waiting on the list, living donors can be used successfully. Intestinal grafts contain $150 \mathrm{~cm}$ of ileum with or without a left lateral liver graft depending on the liver function of the patient. The largest published case series reports that no changes in lifestyle, work habits, or psychologic conditions of the donors were detected after donation (16).

\section{Organ Preservation}

The University of Wisconsin (UW) solution is considered the gold standard for the preservation of all organs of the abdomen. However, many studies have shown that other solutions, such as Celsior and HTK, give results similar to those by the UW solution during ischemic periods up to $8 \mathrm{~h}$ in SBT. On comparing UW and HTK, results for graft survival, initial function, endoscopic appearance, and rate of transplant pancreatitis were found to be similar. HTK gives the advantage of better flushing of the microvasculature due to its low viscosity (17).

\section{Postoperative Management and Complications}

Surgical complications such as wound infection, fistula, bleeding, and dehiscence may cause rejection episodes and opportunistic infections postoperatively. The biggest problem in SBT is graft rejection. Rejection is the main cause of morbidity and mortality. Rejection negatively correlates with the survival of the graft. $50 \%-75 \%$ of recipients experience acute cellular rejection, most commonly in the first 90 days, while chronic rejection (CR) occurs in $15 \%$ of patients (18). Severe rejections in SBT have critical consequences compared with other solid organs, with a mortality rate of $50 \%$.

\section{Immunological Complications}

Compared with other solid organ transplantations, SBT is harder to achieve because small bowel is where most of the immune cells reside (more than $80 \%$ ). Previous studies have shown that the small bowel allograft, particularly the ileal part, is the most vulnerable to AR in frequency and severity when compared with other allografts. It represents the "Achilles heel" of multivisceral transplantation. Besides, within 10 weeks of transplantation, enterocytes inhabit the recipients small bowel, which makes the graft highly chimeric (17). Thus, the presence of patients' lymphocytes within the bowel submucosa may not necessarily reflect rejection, but this bidirectional exchange of immune cells is responsible for GVHD with $7 \%-13 \%$ incidence rate $(18,19)$. A retrospective study analyzed GVHD in terms of incidence, risk factors, and impact on survival (18). Young age, recipients with multi-organ grafts, and splenectomized cases were found to be risk factors for GVHD. The effect of donor T cells in the pathophysiology of GVHD has been studied (18). The study showed that the degree of chimerism derived from donor T cells correlates with clinical course of GVHD. In $64 \%$ of the patients, clinical symptoms of GVHD were presented, and all of them had detectible donor T-cell chimerism. The study showed that all the patients responded 
to increase in immunosuppressive therapy, and three of them died due to sepsis and multi-organ failure.

Another immunological complication is inflammatory bowel disease (IBD)-like disease after transplantation. The incidence of IBD in patients with solid organ transplantation is 10 times more than the expected incidence of IBD in the general population (20). Post-transplant IBD is correlated with cytomegalovirus infection, Epstein-Barr virus infection, post-transplant lymphoproliferative disease, and use of tacrolimus (21). Another possible mechanism can be donor lymphocytes having the genetic information for an abnormal inflammatory response. Further, in colonic mucosa, failure of physiological control by donor-derived regulatory $T$ cells causes intestinal inflammation, which may demonstrate itself as an Arthus-like reaction (22). In a previous study, the use of anti-TNFa showed dramatic clinical and histological improvement in two children (23). Anti-TNFa therapy also has some benefits in treating steroid and thymoglobulin-resistant AR episodes.

In the course of AR, gene expression of TNFa is upregulated soon after transplantation and it increases further (24). This process is associated with immunoregulatory activation and induction of apoptosis and T-cell proliferation (25). Many studies have shown that in patients with refractory $A R$, infliximab can be a good therapeutic option (26). TNFa mRNA expression is slightly elevated $24 \mathrm{~h}$ after transplantation due to ischemia reperfusion injury. On occurrence of $A R, 168 \mathrm{~h}$ after transplantation, the expression levels of TNFa mRNA increase in the intestinal muscular layer (24).

\section{Rejection}

The diagnosis of AR is possible by clinical, endoscopic, and pathological evaluation. The gold standard for the diagnosis of AR is pathologic evaluation by biopsy. Endoscopic observation and biopsies are performed through ileostomy. The endoscopic observation should be performed two to three times in a week in the first three months. Then, it is performed once a month according to the course of transplantation (27). Mucosal erythema, edema, shortened and flattened villi, friability, and ulcerations are endoscopic findings associated with AR. Sensitivity and specificity of endoscopy are 52\% and 93\%, respectively (27). As the lesions may be absent in some of the segments, on suspicion of rejection, several biopsies should be performed.

Local innate immune activation can easily increase the activity of antigen-presenting cells, and this can increase sensitization to donor antigens. To protect grafts against T-cell mediated rejection, lymphocyte-depleting agents are used successfully for induction and long-term tacrolimus with steroids for maintenance treatment. However, as long as AMR it is relatively insensitive to corticosteroids, antibody-mediated rejection (AMR) still is an important problem in SBT (28).

Some authors have successfully performed SBT and multivisceral transplantation across a positive crossmatch (29). Like other solid organ transplantations, donor-specific antibody (DSA) formation in the serum of the patients is associated with AMR. In contrast with preformed DSA, de novo DSA, which is a potent diagnostic marker for $A R$ and $C R$, is associated with adverse clinical outcomes (29). De novo DSAs seem to appear in approximately one-fourth of the patients after transplantation as a result of alloreactive humoral responses and are associated with increased incidence of CR and graft loss. Specimens of the intestinal mucosa do not contain mesenteric vascular structures, and C4d staining is nonspecific. Therefore, histologic findings of AMR in SBT are not yet well-defined (30). AMR is one of the main problems for transplantation in pre-sensitized recipients. Further, DSAs are the most important cause of longterm CR and late allograft failure (31). DSAs can be detected by single antigen fluorescent bead assays via Luminex. The underlying pathophysiology of CR and late allograft failure is mesenteric arteriopathy, which is highly associated with DSA and complement development.

\section{Immune Monitoring}

Immune monitoring is crucial after SBT. Within 5 years after transplantation, approximately $50 \%-75 \%$ patients experience $A R,>10 \%$ patients experience lymphoproliferative diseases due to over immune suppression, especially in children, and $>10 \%$ patients experience $C R$, which results in graft loss (31).

Small bowel transplantation, unlike other types of transplantation, does not have reliable markers to predict rejection. Thus, protocol biopsies and histopathological analysis still remain the gold standard for allograft monitoring for rejection. However, biopsy also has some complications such as perforation and ulceration, especially in the grafts from young donors. Diagnosis cannot be defined in $30 \%$ of biopsies. Therefore, multiple biopsies should be performed to exclude rejection (32). It is recommended to perform systemic biomarker evaluation concomitant with histopathological examination. Among auxiliary assays, increase in use of measurements of citrulline level in the blood, cytofluorographic analysis of peripheral immune cell population, cytokine profiles, and the quantitation of distinct gene set changes have been observed (33-35). Developments in the understanding of genes promise to provide; limited gene sets taken from blood or from intestinal biopsies to enhance pathological diagnosis of rejection.

\section{a. Biomarkers}

Myeloid dentritic cells (MDC) are potent antigen-presenting cells and serve as markers for the recipients who are prone to AR. Plasmacytoid CD123 (PCD) dendritic cells have tolerogenic effects, and they gradually increase in number during the rejection-free post-transplant period. A single-center study conducted with 23 children declares that the children who experienced AR had significantly higher MDCs/PDCs ratios compared with non-rejecters (36). The carboxyfluorescein succinimidyl ester (CFSE) mixed leucocyte response (MLR) detects cytotoxic-T- cell proliferation as a predictor of AR in solid organ transplantation. The ratio of donor and third-party-induced proliferative CFSE T cells, which is measured by flow cytometry, was assessed as the immune reactivity index for each subset. Immune reactivity index score of more than 1 shows increased risk of rejection and that of less than 1 signifies reduced risk. The sensitivity and specificity of the test for predicting AR in intestinal transplantation is $87.5 \%$ and $83.3 \%$, respectively (37).

It has been shown that microRNAs have a critical role in immune regulation. Data suggests that microRNAs have a critical role in the activation of infiltrating cells during AR in SBT (38). These differences in microRNA expression patterns can 
be used to identify new biomarkers and therapeutic targets for immunosuppressive drugs. Wide interpatient variability reduces the ability to set cutoff points for rejection across normal population. Nonetheless, these predictive and discriminative biological markers require further large-scale in-depth studies.

Nucleotide Oligomerization Domain (NOD)-2 is a pattern recognizing receptor and plays an important role in limiting innate immune activation. NOD-2 is found on Paneth cells that sense bacterial residues, dendritic cells, and macrophages. Any malfunction in this sensor may result in the initiation of the rejection process via a misexpression with intestinal antibacterial peptides and other alterations in innate immune responses. Therefore, the immune system being provoked, results in structural shifts seen during rejections. For this reason, suppressing the levels of enterobacteria can be a suitable alternative for the elongation of small intestinal allograft survival. Normally, the recognition of intracellular bacteria controls the antimicrobial peptides secreted by Paneth cells. Patients with NOD-2 polymorphisms who undergo SBT are at significantly greater risk of early rejection, decreased survival, and death due to sepsis. SBT patients who have NOD-2 polymorphisms show higher incidence for early rejections and deaths due to sepsis, and their survival rate may be lower (39).

Besides biomarkers that identify ARs, some markers have been investigated to find out the recipients who are prone to AR attacks. Although these markers have more than $90 \%$ sensitivity and specificity for predicting AR and appear to give promising results, routine monitoring in a clinical setting has not been established.

\section{b. Imaging tests}

Imaging modalities like positron-emission tomography and other radioactive tracers such as ${ }^{111} \mathrm{In}$-labeled platelets, radiolabeled white cell scintigraphy, and MRI have been investigated for predicting AR. However, none of these techniques are useful owing to the low number of SBTs; as the small number does not make it possible to interpret any possible changes.

An animal experiment showed that real-time determinations of fluid or/and electrolyte movement through the small intestine could be obtained by measuring luminal fluid changes using a new modified perfusion system together with FITC-inulin (40). By this way, it can be possible to reliably follow-up any intestinal dysfunction.

Laser Doppler monitoring is another invasive method that can be monitored continuously. During this procedure, which is quite easy to perform, the monitoring device should be attached to the intestine. An implantable Doppler, which is very swift and performs continuous monitoring, appears to be the ideal method for monitoring grafts. Although the implantable Doppler is swift and gives a sensitive screening, its sensitivity may be a little low. The process of placement of the implantable Doppler in the intestine at the vascular pedicle may cause some risks, wherein venous congestion may be induced on the thin walls of the visceral veins; however, by placing the monitoring device around the vein of the transplant, an early warning of venous congestion may be detected.

\section{c. Stool tests}

Recent discoveries about intestinal flora in various diseases may be helpful in studying the alterations in the bowel microflora after transplantation and examining the intestinal allograft damage. Bowel transplant recipients have shown different alterations in their intestinal microflora. In episodes of rejection, the proportions of phylum Firmicutes and the order Lactobacillales have shown a significant decrease, whereas the phylum Proteobacteria, and especially the Enterobacteriaceae, have increased significantly. In such case Firmicutes can be useful to discriminate active rejection from non-rejection (41). The absolute values determined from enterobacteria to total bacteria ratio showed an improvement in detecting differences between healthy transplants and rejections. Thus, a cut-off point of $<49.7 \%$ of Firmicutes would find out an active rejection with $90 \%$ of sensitivity and $90.9 \%$ specificity.

Calprotectin, which is an S-100 protein, released from infiltrating lymphocytes can be estimated by stool testing and these early results seem promising for observing the rejection of intestinal graft prior to the onset of histological changes of AR; wherein calprotectin elevations suggest rejection and normal levels are associated with normal histology. Calprotectin shows up in the fecal content by the migration of neutrophils into the intestine and is a sensitive marker of abnormal activity in inflammatory intestinal diseases (42). It is recommended that the recipients with high levels of calprotectin should undergo intestinal biopsy. Another study showed that recipients with rejection show much higher stool calprotectin levels compared with patients with viral enteritis and those with normal biopsies. This study indicated an optical cutoff level to separate rejections from other diagnoses to be $92 \mathrm{mg} / \mathrm{kg}$ with sensitivity of $83 \%$ and specificity of $77 \%$ (43). Another suggested predictor of rejection is IGF-1. During episodes of intestinal dysfunction, calprotectin levels significantly increase and IGF-1 levels decrease (44). Patients with lowered IGF-1 and raised calprotectin should have enteral feeding interrupted and should be put back on TPN until the cause of high calprotectin levels is determined.

Citrulline is a protein released from enterocytes and levels of citrulline show negative correlation with the function of the small bowel graft (33). From its enterocyte-specific origin, it first gained interest in intestinal failure as a marker. Although diminishing plasma levels of citrulline appear to be associated with mucosal damage, it does not reliably predict rejection. In a recent study, citrulline was assessed as a marker in a patient with a wide variety of intestinal pathologies and lack of a predictor for rejection (44).

Loss of plasma proteins into the gastrointestinal lumen may be detected by the fecal content of alpha- 1 antitrypsin. Increased losses into feces can be caused by inflammatory diseases resulting in enhanced vascular wall permeability, gut erosions causing loss of interstitial fluid, increased venous pressure, and lymphatic obstruction (44). Increased losses into feces may be due to loss of interstitial fluid and rise in venous pressure caused by gut erosions, lymphatic obstruction, and enhanced vascular wall permeability due to inflammatory diseases.

\section{d. Other predictors}

The motility of the transplanted intestine is very important for the sustainability of the transplant. At the first stage, when 
there is no extrinsic signaling, the interstitial cells of Cajal help regulate intestinal motility with their pacemaker function. In case of ischemia, reperfusion, and rejection, immunological and local inflammatory changes in the tunica muscularis of transplanted intestines also cause dysmotility. Therefore, dysmotility can be one of the predictors of AR (45).

Bile acid, serum gentamicin, Granzyme B and perforin, proinflammatory mediator leukotriene $\mathrm{E} 4$, and vitamins B2, B5, and B6 were tested as markers for rejection after SBT, but none of these were found to be sufficiently reliable (46).

\section{Immunosuppressive Therapy}

Several strategies and immunosuppressive regimens were utilized in SBT (27). In most centers, best results were achieved by anti-lymphocyte antibodies and monoclonal or polyclonal induction therapy $(7,27)$. Mostly thymoglobulin, alemtuzumab, basiliximab, and daclizumab are used for this induction. Tacrolimus administration for the maintenance of immune suppression is continued; in the first month, levels are kept as 12-15 $\mathrm{ng} / \mathrm{mL}$ and lowered to $8-12 \mathrm{ng} / \mathrm{mL}$ after the first stage (10). As in other abdominal organ transplants, corticosteroids are also used, and removed in accordance with the type of grafts and preference of each center.

Although some improvement has been achieved in controlling rejection after SBT with new immune suppressants, ARs and CRs still show a high rate of occurrence. Therefore, some novel attempts, such as bone marrow mesenchymal stem cell (BMMSC) transplantation in addition to SBT, are being examined $(47,48)$. BMMSCs, which show an immunosuppressive activity in transplantation, are detected for stopping the immunological refractory cells that attack the transplanted organs. They can also urge and carry on the process of epithelialization of the small intestinal epithelium. An animal experiment showed that the immunoregulatory effect of these cells (BMMSC) was affected by the balance of Th1/Th2, Th17/ Treg, the related cytoxines, NK-cell activity, and Treg expansion and that these cells prevented AR in SBT (48). Tolerogenic regiments that provoke Tregs and chimerism and block the development of DSA are future treatment goals to be reached. Current studies have shown that intestinal mucosa repair is enhanced by cell proliferation and/or inhibition of epithelial cell apoptosis, which is enhanced by the BMMSC synthesis. In this setting the released cytokines and growth factors are factors as interleukin-11 hepatocyte growth factor, fibroblast growth factor-2 and insulin-like growth factor-I. Some beneficial effects of BMMSC transplantation with SBT have been shown in clinical settings (47).

\section{Tissue Engineering}

Current trials have shown that progress in the description and propagation of small bowel stem cells and tissue engineering developments promise that realistic alternatives to deceased donors can be seen in the future. In an animal model, small sections of small bowel, produced by implanting intestine stem cells on collagen structures, demonstrated improved growth after placing the graft in continuity with remnant bowel surgically (49). Progress in experimental trials have shown that efforts in the future would be toward the amelioration of injured bowels or the trials of creating new intestinal tissues from autologous stem cells.

\section{CONCLUSION}

As a conclusion of these developments, morbidity and mortality rates of SBT have decreased, lately. As the experience of the centers increase and the mechanisms of immune alloreactivity are elucidated, authors believe that the success in this field will be enhanced. Stem cell transplantation and tissue engineering are seen as promising procedures for the future.

Peer-review: Externally peer-reviewed.

Conflict of Interest: No conflict of interest was declared by the author.

Financial Disclosure: The author declared that this study has received no financial support.

\section{REFERENCES}

1. Grant D, Abu-Elmagd K, Mazariegos G, Vianna R, Langnas A, Mangus $R$, et al. Intestinal transplant registry report: global activity and trends.; Intestinal Transplant Association. Am J Transplant 2015; 15: 210-219.[CrossRef]

2. Gotthardt DN, Gauss A, Zech U, Mehrabi A, Weiss KH, Sauer P, et al. Indications for intestinal transplantation: recognizing the scope and limits of total parenteral nutrition. Clin Transplant 2013; 27 Suppl 25: 49-55. [CrossRef]

3. Abu-Elmagd KM. The small bowel contained allografts: existing and proposed nomenclature. Am JTransplant 2011; 11: 184-185. [CrossRef]

4. Smith JM, Skeans MA, Horslen SP, Edwards EB, Harper AM, Snyderf JJ, et al. OPTN/SRTR 2013 Annual Data Report: intestine. Am J Transplant 2015; 15 Suppl 2: 1-16. [CrossRef]

5. Kaufman SS, Pehlivanova M, Fennelly EM, Rekhtman YM, Gondolesi GE, Little CA, et al. Predicting liver failure in parenteral nutrition-dependent short bowel syndrome of infancy. J Pediatr 2010; 156: 580-585. [CrossRef]

6. Berney T, Kato T, Nishida S, Tector AJ, Mittal NK, Madariaga J, et al. Portal versus systemic drainage of small bowel allografts: comparative assessment of survival, function, rejection, and bacterial translocation. J Am Coll Surg 2002; 195: 804-813. [CrossRef]

7. Fryer JP. The current status of intestinal transplantation. Curr Opin Organ Transplant 2008; 13: 266-272. [CrossRef]

8. Kato T, Selvaggi, Gaynor JJ, Takahashi H, Nishida S, Moon J, et al. Inclusion of donor colon and ileocecal valve in intestinal transplantation. Transplantation 2008; 86: 293-297. [CrossRef]

9. Suzuki H, Li XH, Miyamoto M, Sano T, Hattori Y, Yamashita A. Induction of transplantation tolerance in adult rats by vascularized spleen transplantation. Transplantation 1997; 64: 650-654. [CrossRef]

10. Kato T, Tzakis AG, Selvaggi G, Gaynor JJ, Takahashi H, Mathew J, et al. Transplantation of the spleen: effect of splenic allograft in human multivisceral transplantation. Ann Surg 2007; 246: 436-446. [CrossRef]

11. Upile T, Jerjes W, El Maaytah M, Hopper C, Searle A, Wright A. Direct microvascular monitoring of a free autologous jejunal flap using microendoscopy: a case report. BMC Ear Nose Throat Disord 2006; 6: 14. [CrossRef]

12. Sheth J, Sharif K, Lloyd C, Gupte G, Kelly D, de Ville de Goyet J, et al. Staged abdominal closure after small bowel or multivisceral transplantation. Pediatr Transplant 2012; 16: 36-40. [CrossRef]

13. Watson MJ, Kundu N, Coppa C, Djohan R, Hashimoto K, Eghtesad $B$, et al. Role of tissue expanders in patients with loss of abdominal domain awaiting intestinal transplantation. Transpl Int 2013; 26: 1184-1190. [CrossRef]

14. Gerlach UA, Pascher A. Technical advances for abdominal wall closure after intestinal and multivisceral transplantation. Curr Opin Organ Transplant 2012; 17: 258-267. [CrossRef]

15. Cobianchi L, Zonta S, Vigano J, Dominioni T, Ciccocioppo R, Morbini $\mathrm{P}$, et al. Experimental small bowel transplantation from 
non-heart-beating donors: a large-animal study. Transplant Proc 2009; 41: 55-56. [CrossRef]

16. Gangemi A, Tzvetanov IG, Beatty E, Oberholzer J, Testa G, Sankary $\mathrm{HN}$, et al. Lessons learned in pediatric small bowel and liver transplantation from living-related donors. Transplantation 2009; 87: 1027-1030. [CrossRef]

17. Oltean M, Churchill TA. Organ-specific solutions and strategies for the intestinal preservation. Int Rev Immunol 2014; 33: 234-244. [CrossRef]

18. Mathew JM, Tryphonopoulos P, DeFaria W, Ruiz P, Miller J, Barrett $\mathrm{TA}$, et al. Role of innate and acquired immune mechanisms in clinical intestinal transplant rejection. Transplantation. 2015; 99: 1273-1281. [CrossRef]

19. Shin CR, Nathan J, Alonso M, Yazigi N, Kocoshis S, Tiao G, et al. Incidence of acute and chronic graft-versus-host disease and donor T-cell chimerism after small bowel or combined organ transplantation. J Pediatr Surg 2011; 46: 1732-1738. [CrossRef]

20. Hampton DD, Poleski MH, Onken JE. Inflammatory bowel disease following solid organ transplantation. Clin Immunol 2008; 128: 287-293. [CrossRef]

21. Godt C, Regnery A, Schwarze B, Junker K, Porschen R. A rare cause of ulcerative colitis - diarrhoea and perianal bleeding due to posttransplant lymphoproliferative disorder (PTLD). Z Gastroenterol 2009; 47: 283-287. [CrossRef]

22. Actis GC, Rosina F, MacKay IR. Inflammatory bowel disease: beyond the boundaries of the bowel. Expert Rev Gastroenterol Hepatol 2011; 5: 401-410. [CrossRef]

23. De Greef E, Avitzur Y, Grant D, De-Angelis $\mathrm{M}, \mathrm{Ng} \mathrm{V}$, Jones $\mathrm{N}$, et al. Infliximab as salvage therapy in paediatric intestinal transplant with steroid- and thymoglobulin-resistant late acute rejection. $J$ Pediatr Gastroenterol Nutr 2012; 54: 565-567. [CrossRef]

24. Schaefer N, Tahara K, Websky MV, Koscielny A, Pantelis D, Kalff JC, et al. Acute rejection and the muscularis propria after intestinal transplantation: The alloresponse, inflammation, and smooth muscle function. Transplantation 2008; 85: 1465-1475. [CrossRef]

25. Adams AB, Larsen CP, Pearson TC, Newell KA. The role of TNF receptor and TNF superfamily molecules in organ transplantation. Am J Transplant 2002; 2: 12-18. [CrossRef]

26. Pirenne J, Kawai M. Intestinal transplantation: Evolution in immunosuppression protocols. Curr Opin Organ Transplant 2009; 14: 250-255. [CrossRef]

27. Lauro A, Pinna AD, Pellegrini S, Bagni A, Zanfi C, Dazzi A, et al. Long-term endoscopic follow-up in small bowel transplant recipients: single-center series. Transplant Proc 2014; 46: 2325-8. [CrossRef]

28. Archdeacon P, Chan M, Neuland C, Velidedeoglu E, Meyer J, Tracy $\mathrm{L}$, et al. Summary of FDA antibody-mediated rejection workshop. Am J Transplant 2011; 11: 896-906. [CrossRef]

29. Gerlach UA, Lachmann N, Sawitzki B, Arsenic R, Neuhaus $P_{,}$ Schoenemann $C$, et al. Clinical relevance of the de novo production of anti-HLA antibodies following intestinal and multivisceral transplantation. Transpl Int 2014; 27: 280-289. [CrossRef]

30. Tsai HL, Island ER, Chang JW, Gonzalez-Pinto I, Tryphonopoulos P, Nishida S, et al. Association between donor-specific antibodies and acute rejection and resolution in small bowel and multivisceral transplantation. Transplantation 2011; 92: 709-715. [CrossRef]

31. Nayyar N, Mazariegos G, Ranganathan S, Soltys K, Bond G, Jaffe $\mathrm{R}$, et al. Pediatric small bowel transplantation. Semin Pediatr Surg 2010; 19: 68-77. [CrossRef]

32. Wu T, Abu-Elmagd KM, Bond G, Nalesnik MA, Randhawa P, Demetris AJ. A schema for histologic grading of small intestine allograft acute rejection. Transplantation 2003; 75: 1241-1248. [CrossRef]

33. Mercer DF. Hot topics in postsmall bowel transplantation: noninvasive graft monitoring including stool calprotectin and plasma ci- trulline. Curr Opin Organ Transplant 2011; 16: 316-322. [CrossRef]

34. Sageshima J, Ciancio G, Gaynor JJ, Chen L, Guerra G, Kupin W, et al. Addition of anti-CD25 to thymoglobulin for induction therapy: delayed return of peripheral blood CD25-positive population. Clin Transplant 2011; 25: E132-135. [CrossRef]

35. Asaoka T, Island ER, Tryphonopoulos P, Selvaggi G, Moon J, Tekin $A$, et al. Characteristic immune, apoptosis and inflammatory gene profiles associated with intestinal acute cellular rejection in formalin-fixed paraffin-embedded mucosal biopsies. Transpl Int 2011; 24: 697-707. [CrossRef]

36. Gupta A, Ashokkumar C, Ningappa M, Sun Q, Higgs BW, Snyder S, et al. Elevated myeloid: plasmacytoid dendritic cell ratio associates with early acute cellular rejection in pediatric small bowel transplantation. Transplantation. 2010; 89: 55-60. [CrossRef]

37. Ashokkumar C, Gupta A, Sun Q, Higgs BW, Ningappa M, Snyder $\mathrm{S}$, et al. Proliferative alloresponse of $\mathrm{T}$ cytotoxic cells identifies rejection-prone children with small bowel transplantation. Transplantation 2010; 89: 1371-1377. [CrossRef]

38. Asaoka T, Sotolongo B, Island ER, Tryphonopoulos P, Selvaggi $G$, Moon J, et al. MicroRNA signature of intestinal acute cellular rejection in formalin-fixed paraffin-embedded mucosal biopsies Am J Transplant 2012; 12: 458-468. [CrossRef]

39. Ningappa $M$, Higgs BW, Weeks DE, Ashokkumar C, Duerr RH, Sun Q, et al. NOD2 gene polymorphism rs2066844 associates with need for combined liver intestine transplantation in children with short-gut syndrome. Am J Gastroenterol 2011; 106: 157-165. [CrossRef]

40. Munoz-Abraham AS, Judeeba S, Alkukhun A, Alfadda T, PatronLozano R, Rodriguez-Davalos $\mathrm{Ml}$, et al. A new method to measure intestinal secretion using fluorescein isothiocyanate-inulin in small bowel of rats. J Surg Res 2015; 197: 225-230 [CrossRef]

41. Oh PL, Martínez I, Sun Y, Walter J, Peterson DA, Mercer DF. Characterization of the ileal microbiota in rejecting and nonrejecting recipients of small bowel transplants. Am J Transplant 2012; 12: 753-762. [CrossRef]

42. Lorenz F, Marklund S, Werner M, Palmqvist R, Wahlin BE, Wahlin A. Fecal calprotectin as a biomarker of intestinal graft versus host disease after allogeneic hematopoietic stem cell transplantation. Sci Rep 2015; 5: 7920. [CrossRef]

43. Sudan D, Vargas L, Sun Y, Bok L, Dijkstra G, Langnas A. Calprotectin: a novel noninvasive marker for intestinal allograft monitoring. Ann Surg 2007; 246: 311-315. [CrossRef]

44. Vrakas G, O'Connor M, Matsou A, Kwon Y, Li A, Guerra J, et al. Markers of malnutrition after intestinal transplantation: the role of IGF-1 and calprotectin. J Clin Biochem Nutr 2015; 56: 64-65. [CrossRef]

45. von Websky MW, Kalff JC, Schäfer N. Current knowledge on regulation and impairment of motility after intestinal transplantation. Curr Opin Organ Transplant 2015; 20: 303-307. [CrossRef]

46. Girlanda R, Cheema AK, Kaur P, Kwon Y, Li A, Guerra J, et al. Metabolomics of human intestinal transplant rejection. Am J Transplant. 2012; 12 Suppl 4: 18-26. [CrossRef]

47. Doğan SM, Kılınç S, Kebapçı E, Tuğmen C, Gürkan A, Baran M, et al. Mesenchymal stem cell therapy in patients with small bowel transplantation: Single center experience World J Gastroenterol 2014; 20: 8215-8220. [CrossRef]

48. Houchen CW, George RJ, Sturmoski MA, Cohn SM. FGF-2 enhances intestinal stem cell survival and its expression is induced after radiation injury. Am J Physiol 1999; 276: G249-G258.

49. Sala FG, Kunisaki SM, Ochoa ER, Vacanti J, Grikscheit TC. Tissueengineered small intestine and stomach form from autologous tissue in a preclinical large animal model. J Surg Res 2009; 156: 205-212. [CrossRef] 